\title{
Estado de la cuestión de la experiencia docente en los procesos de inclusión de alumnos inmigrantes centroamericanos en
} Tapachula, Chiapas

\author{
Mauricio Zacarías Gutiérrez \\ Recebido em: 20/10/2014. Aprovado em: 17/11/2014. Disponibilizado em: 26/12/2014
}

1. Mauricio Zacarías Gutiérrez és Estudiante del Doctorado en Estudios Regionales. Universidad Autónoma de Chiapas. México. mazag@hotmail.com

\section{Resumen}

En el presente estudio se problematiza el objeto de estudio la experiencia docente en los procesos de inclusión de niños y niñas inmigrantes centroamericanos en el contexto del municipio de Tapachula, Chiapas. Para abordar el objeto se parte de la cuestión que la educación ha sido objeto de estudio de disciplinas como la economía, la política, la sociología, la pedagogía, entre otros, los cuales han aportado concepciones teóricas de cómo la entienden, por lo que cabe decir que la educación es un campo amplio que no se le puede definir de manera llana, que al ser tomada como objeto de estudio, se le ha explicado, interpretado y se ha generado cambio, en este sentido quien investiga en la educación, puede descubrir fenómenos que en la vida cotidiana no se ven, sino que sólo se viven, y corresponde al investigador de la educación asombrarse lo que la cotidianidad mantiene. Lo anterior lleva a que se problematice el estado de la cuestión sobre lo que se ha escrito de la educación como derecho, desde dos ámbitos: el primero referido a la inclusión educativa en el ámbito de las necesidades educativas especiales para personas que presentan una discapacidad física o mental, así como investigaciones que se han desarrollado entorno a la interculturalidad; el segundo a la educación como derecho de la población migrante en México.

Palabras clave: derecho a la educación, inmigración, escuela.

\footnotetext{
Abstract

In the present study the subject matter of teaching experience in the processes of inclusion of children and Central American immigrant girls in the context of the town of Tapachula, Chiapas is problematized. To address the object is part of the question that education has been studied in disciplines such as economics, politics, sociology, pedagogy, among others, which have provided theoretical conceptions of how to understand it, so it fits say that education is a broad field that he can not be defined in plainly, that being taken as an object of study, has been explained, interpreted and generated change in this regard who researches in education, you may find phenomena in everyday life are not seen, but only live, and corresponds to education researcher wonder what keeps everyday. This leads to the state of affairs on what has been written education as a right, from problematice two areas: the first refers to inclusive education
} 
in the field of special needs education for people with a physical disability or mental, as well as investigations that have developed around multiculturalism; the second to education as a right of migrants in Mexico.

Keywords: rights to education, immigration, school

GUTIÉRREZ, M.Z.: Estado de la cuestión de la experiencia docente en los procesos de inclusión de alumnos inmigrantes centroamericanos en Tapachula, Chiapas 



\section{Introducción}

1.1 Sobre la discusión hecha en investigaciones entorno a la inclusión educativa

Investigaciones sobre Inclusión Educativa, se han realizado en México, y en otros países. La manera en que se ha abordado el tema es diverso, no hay linealidad que especifique cómo hacerlo. Lo importante de estas investigaciones es que permiten reconocer de dónde parten, cómo abordan la problemática, qué discusiones abrieron y que abonaron a la investigación realizada.

Revisando estados de la cuestión respecto a la inclusión educativa, se encuentra la investigación que realizan Alcántara Santuario y Navarrete Cazales (2014), quienes centran su objeto de estudio de la inclusión a la educación desde los espacios universitarios que se han abierto para los grupos indígenas en el Estado mexicano, enfatizan el apoyo que ha dado el gobierno federal para su consolidación y los avances cuantitativos que se han hecho en la materia. Consideran que la inclusión se ha dado paulatinamente en el ingreso de las personas pertenecientes a un grupo indígena en las universidades creadas para ellos, aunque no específicamente para ellos según una cita d Shmelkes dentro del mismo texto, sino para todo aquel que solicite en esas universidades un espacio para formación profesional. Sin embargo la investigación se centra en el análisis de la poca inserción que tienen los alumnos indígenas en las Universidades e institutos de educación superior estatales o federales. Ellos asumen a partir de la investigación realizada que la poca inserción al nivel superior se debe a la memoria cultural de estos alumnos.

En ot, e solo hay una mínima parte o más bien frase de lo que es la atención a estos alumnos y su condición), sino el enfoque es planteado en apoyar a los jóvenes que por diversas razones abandonaron la escuela, a partir del programa Escuelas de Reingreso, en el cual se centran en estudiar ra perspectiva, la investigación de Montes y Ziegler (2010), se centra en el estudio de la inclusión desde una perspectiva de apoyar a los alumnos vulnerables, que no terminaron la educación secundaria, en Buenos Aires, Argentina. Por lo que no se considera a los inmigrantes, (claro la experiencia que han tenido los alumnos que abandonan la educación secundaria regular y se incorporan a esta.

La manera en que plantean la inclusión educativa o la forma en que lo conceptualizan los autores en la investigación es que:

La demanda social y normativa por la inclusión y la obligatoriedad tensiona lo conocido y provoca la necesidad de experimentar otras formas de enseñanza y de entrar en diálogo con los jóvenes en general y, en particular con los más vulnerables, en términos de sus trayectorias de vida (Montes y Ziegler, 2010, p. 1090).

El argumento de los autores, considera que la inclusión educativa para los alumnos vulnerables debe considerarse más allá de la norma establecida, es decir, abrir la posibilidad de tener otras formas de enseñanza. Considerando, que no solo es cuestión de proponer un programa alterno como el de Escuelas de Reingreso, sino entender la complejidad del asunto en el que están los alumnos.

Por otro lado, el documento que publica la Secretaría de Educación Pública (SEP) (2012) Buenas Prácticas de Educación Inclusiva, se plasman experiencias de profesores que han trabajado con alumnos que presentan una discapacidad física $o$ cognitiva. En ella los profesionales en el área de la Necesidad Educativa Especial dan cuanta del apoyo que brindan a los que 
requieren de su asistencia, cómo es que sensibilizan a la sociedad y la sociedad responde a lo que ellos están planteando.

El objetivo del documento se centra en el proceso que realizan con los alumnos que presentan una discapacidad física o cognitiva, no lo nombran inclusión educativa, sino integración educativa, dado que se consideran que los alumnos que presentan una necesidad física o cognitiva deben contar con el apoyo de personal profesionalizado en el área, más el apoyo de los profesores que los atienden dentro de las aulas regulares, de los padres de familia, de amigos, de los compañeros del aula, entre otros (SEP, 2012), para que desarrollen habilidades, destrezas y se integren a la sociedad.

Las experiencias de atención a niños que presentan una discapacidad física o cognitiva, que publica la SEP en México, se alinea a lo que se norma para los niños que presentan Necesidades Educativas Especiales, en la Declaración de Salamanca y Marco de Acción para las necesidades Educativas especiales (Organización de las Naciones Unidas para la Educación, la Ciencia y la Cultura, 1994). Al respecto sobre las necesidades educativas especiales, dice la Declaración que: "En el contexto de este Marco de Acción el término "necesidades educativas especiales" se refiere a todos los niños y jóvenes cuyas necesidades se derivan de su capacidad o sus dificultades de aprendizaje" (1994, p. 15).

La cita permite considerar que las necesidades educativas especiales la están refiriendo a los alumnos que presentan una discapacidad física o cognitiva, en el cual la escuela como integradora juega un protagónico, de ahí que las experiencias que compila la SEP, den muestra de estas escuela integradoras y cómo los profesionales del área se integran con los profesionales de educar a los niños a una educación completa, en este sentido la Declaración de Salamanca dice que: "El principio fundamental que rige las escuelas integradoras es que todos los niños deben aprender juntos, siempre que sea posible, haciendo caso omiso de sus dificultades y diferencias" (UNESCO, 1994, P. 11).

\section{Una idea integradora}

Se tiene una idea integradora desde la escuela y de quienes participan directamente en ellas: alumnos, profesores, padres de familia, directivos, entre otros. ¿Qué implicaciones ha tenido ello en las escuelas regulares del país donde hay alumnos que presentan una necesidad física y/o cognitiva y hay alumnos inmigrantes? ¿Las escuelas han tenido que aprender a salir de lo uniforme y dar paso a la diversidad? ¿En México tenemos escuelas inclusivas en atención a alumnos que presentan una situación cognitiva y/o física, condiciones de vulnerabilidad y condiciones de migración?, ¿cómo son las escuelas que atienden a alumnos que se encuentran en situación de vulnerabilidad, migración o desventaja social?, ¿cómo se dan los procesos de inclusión en estos espacios? ¿Cómo intervienen los alumnos, los profesores, los padres de familia, los directivos, entre otros, dentro de su proceso e integración e inclusión dentro de la escuela en los espacios donde se encuentran altos índices de alumnos provenientes de otros países?

Ante los cuestionamientos, cabe mencionar lo siguiente de la Declaración de Salamanca "la integración de niños con discapacidades deberá formar parte de los planes nacionales de "Educación para todos"' (UNESCO, 1994, p. 24). El posicionamiento de la Declaración, de incluir a todos en la escuela, es holístico, desde un plan nacional, sin embargo no deja de ser un posicionamiento desde el otro que tiene que integrarse, falta considerar que somos diferentes y sobre esa diferencia la sociedad se construye. 
En atención de lo que plantea la Declaración de Salamanca, de que la integración de los alumnos con discapacidades debería formar parte de los planes nacionales, en México se ha atendido la sugerencia, sin embargo, el posicionamiento de la misma ha sido como lo planteaba la declaración, de integrar al alumno con discapacidad, aunque ha habido avances en la legislación educativa en México de pasar de la integración a la inclusión, en la acción la escuela aún no se considera como "una comunidad de aprendizaje compartido [donde] el profesorado va a la escuela a aprender de sus alumnos y con sus alumnos y no a defenderse de los mismos" (López Melero, 2004, p. 101).

El planteamiento de López Melero, se enfoca a incluirnos no desde la política como mandato de Estado nacional, sino como necesidad de persona que se reconoce con otra persona y que juntos creamos los espacios que permiten una mejor conviviencia y desarrollo social. El atrevimiento a pensar de esa manera es que es análogo a la composición del cuerpo humano, de que son muchos factores que influyen a que el organismo de cada persona reaccione de distinta manera ante asombros que lleva en su cotidianidad, por lo que pensar una escuela donde todos aprendemos de todos y estemos conviviendo, no es en el sentido de mandato como idea de vivir en la democracia política, sino en la idea de una democracia donde las personas nos reconocemos desde la diversidad que poseemos.

A esta idea, se suma el planteamiento que tienen Casado Muñoz y Lezcano Barbero (2012) que la inclusion educativa va mas allá de una mera integracion de los alumnos diferentes al aula. En el entendido que la diferencia va desde los alumnos que tienen necesidades educativas especiales, los de color, pertencer a una raza, a una etnia, vivir en vulnerabiidad, ser inmigrantes, entre otros.
Cuestión que Aja (2000) plantea en la recuperacion que hace de la educacion inclusiva en España.

Casado Muñoz y Lezcano Barbero (2012) dicen que los conceptos integracion e inclusión se han usado como sinónimos en un tiempo y pareciera que se usan para nombrar lo mismo, sin embargo la situación es diferente, pues desde el modo en que analizan la integracion, es esa donde se integra al alumno para que se adapte al status quo del país, la inclusión la sitúan a la adaptación que hace la escuela para los alumnos que atiende.

Si se toma como válido que el proceso de inclusión es la adaptación del alumno a la escuela, hay que repensar esa manera de nombrarlo, Opperti al respecto dice que "repensar la inclusión es repensar el sistema educativo. Lo que mide la calidad no es la calidad de escuelas o de inversión, es si se le da a cada alumno una posibilidad personalizada de aprender en un marco colectivo de aprendizaje" (Opertti, 2014). Es entonces considerar o ir amarrando ideas de que la inclusión no es integrar al otro desde donde se encuentra sino tomarlo como otro que es diferente y que contribuye a mi proceso de formativo, como yo influyo en el de él y el de los otros.

Los estudios sobre la inclusión de los alumnos en la escuela, se nombra como inclusión o como integración, la posición que tienen los autores a quienes se revisó depende de su referencia teórica sobre el tema. Sin embargo lo importante de la revisión del estado de la cuestión entorno a la inclusión educativa, permite reconocer cómo se ha hecho un proceso incluir o de integrar, cómo se norma la inclusión desde la declaración de Salamanca y cómo se ha venido considerando la Necesidad Educativa Especial más allá de presentar una situación física o cognitiva para la apropiación del conocimiento científico y el desarrollo social, sino que involucra la 
atención a las personas que por su condición social también se consideran con necesidades educativas especiales.

En el siguiente apartado se hace una revisión del estado de la cuestión de la educación como derecho a todas las personas, enfatizando en la educación a migrantes. Se revisan investigaciones educativas que se han realizado en la migración de población mexicana hacia Estados Unidos y migración centroamericana hacia México.

\section{E1 derecho a la educación de la población infantil migrante}

En el apartado anterior se presentó la cuestión de cómo se ha estudiado la inclusión educativa desde el acceso de los alumnos indígenas a la educación, los que presentan una discapacidad física o cognitiva, los que se encuentran en condiciones vulnerables, y la diferencia que hay entre nombrar inclusión e integración. Ello permitió comprender cómo se ha entendido la inclusión educativa desde estas problemáticas y llevó a la revisión del estado de la cuestión sobre la educación de los migrantes.

En la revisión del estado de la cuestión sobre la inclusión educativa desde la migración se concretiza el objeto de estudio que se estudia de este gran mapa que es la educación, hasta limitarse a estudiar la experiencia de los docentes en los procesos de inclusión de niños y niñas inmigrantes centroamericanos, lo que justifica su importancia a partir de las indagaciones que se hicieron para problematizar dicho objeto.

A continuación se presenta el estado de la cuestión sobre investigaciones que han abordado estudios sobre educación y migración en las fronteras de México: norte y sur. De ellas se identifican perspectivas en que se desarrollan los estudios: legislación, política educativa, exclusión escolar, situación social. A partir de estas perspectivas los autores analizados aportan sobre el derecho a la educación de la población migrante mexicana en Estados Unidos y centroamericana en México. Ello permite reconocer dos cosas: lo que se ha hecho en materia educativa desde la legislación y la política educativa, y la manera en que se excluye a los que menos tienen dentro de la educación y la vulnerabilidad como principal detonador de que el derecho a la educación no se cumpla.

Lo anterior empieza a cuestionar ¿Qué ha implicado para los docentes el derecho a la educación? ¿Cómo han concebido el derecho a la educación? ¿Cómo se ha pensado el profesor en torno al derecho a la educación que tiene el alumno en general? ¿Qué significará para el profesor de educación básica atender el derecho a la educación de la población migrante?, cuestionamientos singulares serían, ¿cómo han vivido el proceso de inclusión de los alumnos provenientes del extranjero los profesores de educación primaria en la región de Tapachula, Chiapas? ¿Qué es para el padre de familia el derecho a la educación?, ¿cómo hace valer ese derecho a su hijo? Para el alumno sería ¿Cómo accede a su derecho a la educación el alumno migrante?

A partir de este entramado de preguntas, se comenta que es multifacético lo que se vive, se siente y se comunica en la escuela, pues los saberes pensados o no, dan sentido a lo que se realiza en el aula y en la escuela. Se da vida a la cotidianidad a partir de la experiencia, Fontana y Urquiza, dicen que se podría "pensar la experiencia escolar, sin demasiadas precisiones, como el modo de habitar la escuela de un ser que existe (2011, p.87).

En otra dimensión problematizadora de las experiencias vividas, se encuentra la investigación de Ayala Carabajo, el estudio se 
centra en cómo han vivido los educadores la esperanza pedagógica por los niños o jóvenes a quienes educan, y "describe el intento de acceder a este mundo experiencial donde reside la esperanza de los educadores por los niños y jóvenes de quienes son responsables, así como el esfuerzo llevado a cabo con el fin de desvelar su importancia y sentido pedagógicos" (2011, p. 119). El estudio lo realizó desde el enfoque cualitativo, ocupando el método fenomenológico que propone Van Manen.

Por otro lado el estudio realizado por Aguilar Nery (2004). Hacia una memoria argumental sobre la educación intercultural en México. Una narrativa desde la frontera norte. Se presenta un análisis de la educación intercultural desde cómo lo está atendiendo y entendiendo el discurso oficial y cómo lo entienden los docentes que trabajan con alumnos indígenas. Que para el caso se habla de la interculturalidad, desde la posición en la inclusión del niño inmigrante indígena en el estado de Baja California.

La investigación de Martínez M. (2011) $¿$ Es el multiculturalismo bueno para los inmigrantes?, presenta una reflexión crítica sobre trabajos que se han elaborado en relación a la inclusión del inmigrante. El planteamiento es estructural y tiene que ver con esta cuestión de la desigualdad, racismo sistémico, la división del trabajo o la normalización. Discute sobre esta educación normalizada que se tiene en los países a donde llega el inmigrante. El posicionamiento abona a la problematización en la cuestión de que no solo cómo se considera el inmigrante en la estructura jurídica política del país, sino en ese discurso del currículo que norma la educación en México a partir de la política educativa.

La investigación de Cárdenas Leitón (2011). Referentes teóricos y metodológicos de los docentes en servicio con una perspectiva de inclusión educativa: creencias y prácticas. Aborda la inclusión educativa a partir del trabajo que realizan los profesores en la atención con niños que presentan una discapacidad física y la formación profesional que poseen (los profesores), por lo que el estudio analiza las creencias, las ideologías de los docentes, a la vez la adecuación curricular y los fundamentos legales en materia de inclusión educativa con niños que tienen necesidades educativas especiales.

El estudio de Cárdenas Leitón permite tomar distancia de que la inclusión que se abordará en este estudio se dimensiona desde lo pedagógico, pues aunque los niños que provienen de Centroamérica, pudieran no tener una discapacidad física, sí existe o están presentando una necesidad de atención a su situación social y cultural de procedencia.

En el proceso de ir clarificando el objeto de estudio, de las experiencias docentes en los procesos de inclusión, desde el estado de la cuestión permite reflexionar la experiencia docente con la inclusión educativa como derecho humano, y lleva a situarnos en la participación del gobierno mexicano en la garantía de los migrantes en el país, pues en los tratados internacionales donde México participa le recomiendan cómo actuar en relación a la protección de ellos. En este sentido se argumenta que los estados nación en el momento de pactar, gozan de restricciones, una de ellas es, no atentar contra los derechos de las personas que dejan su lugar de origen (Kymlicka, 2006).

\section{Igualdad de derechos de todos}

La educación, al ser un derecho para todos, es inclusiva, pero, ¿en México cómo se atiende este asunto?, ¿qué es una educación inclusiva? En esta idea de educación inclusiva "implica que todos los niños y las niñas de una determinada comunidad aprendan juntos 
independientemente de sus condiciones personales, sociales o culturales" (Delgado Montoya, 2007, pág. 51).

En esta idea de aprender juntos, la Constitución Política de los Estados Unidos Mexicanos, sobre los derechos humanos y sus garantías, establece en el artículo primero, referido a los derechos humanos, que "las normas relativas a los derechos humanos se interpretarán de conformidad con esta Constitución y con los tratados internacionales de la materia favoreciendo en todo tiempo a las personas la protección más amplia" (Constitución de los Estados Unidos Mexicanos, 2013, pág. 1) y en materia educativa en el artículo tercero fracción II, apartado c), dice que

[la educación] contribuirá a la mejor convivencia humana, a fin de fortalecer el aprecio y respeto por la diversidad cultural, la dignidad de la persona, la integridad de la familia, la convicción del interés general de la sociedad, los ideales de fraternidad e igualdad de derechos de todos, evitando los privilegios de razas, de religión, de grupos, de sexos o de individuos (Constitución de los Estados Unidos Mexicanos, 2013, pág 4).

El marco legal de la educación en México, apoya en la profundización de la investigación, al abrir la interrogante en relacion al quehacer docente de los profesores, pues ellos, ¿cómo configuran sus experiencias docentes en los procesos de inclusión de niños y niñas inmigrantes centroamericanos en las escuelas primarias públicas de Tapachula, Chiapas?, ¿cuáles son las experiencias docentes respecto a los procesos de inclusión de niños y niñas inmigrantes centroamericanos en las escuelas primarias públicas de Tapachula, Chiapas?

Al considerarse la postura de la garantía de los migrantes en el sistema federal de México, y de otros planteamientos que se han nombrado en la problematización, da pie a desentrañar lo que se da por hecho de que el profesor incluye, pues ¿quiénes son los profesores de educación primaria que laboran en las escuelas primarias ubicadas en Tapachula?, ¿cómo han sido los procesos de formación de los docentes respecto a la inclusión educativa?, ¿qué experiencias tienen los docentes respecto a los procesos de inclusión educativa de niños y niñas inmigrantes?, a partir de sus experiencias como docentes ¿qué prácticas pedagógicas han considerado los docentes para promover la inclusión educativa de niños inmigrantes a partir de la experiencia docente?

No hay una sola respuesta a los cuestionamientos anteriores, implica otros elementos, que en lo que sigue del documento más que aclararse una respuesta, es plantear otras. ¿Por qué se plantea este argumento? Porque el derecho a la educación ha sido un proceso histórico en el devenir de la humanidad que ha hecho historia, por tomar una primer idea sería el surgimiento de la escuela en Prusia, (Doin, 2012) y su propagación por el mundo. En este devenir la educación que da la escuela se ha planteado como derecho, el cual ha sido un proceso en el que ha intervenido no solo el estado como regulador de la sociedad sino también el capitalismo que gradualmente o no, se ha instaurado en la dinámica social.

La educación al convertirse en derecho, que esta salvaguardado más allá de lo interno de un Estado nación, se plantea desde las esferas internacionales, por lo cual los estados nacionales han firmado pactos y acuerdos para que la educación básica sea accesible para todos los menores de edad. Este es el texto que presenta Azúa Reyes (1987), quien centra su atención en analizar cómo se ha pensado el derecho a la educación desde lo racional y social.

En el ámbito de lo racional convergen desde el punto de vista jurídico "la legislación internacional, constituciones políticas de los 
países y documentos de la iglesia católica" (1987, p. 362). En el acto racional, se traspasa el culto, la vida en el clan y en el seno de la familia, que lleva a las personas a un acceso de conocimiento que supere lo experiencial que posean, que los lleve a configurar la razón a partir del conocimiento cotidiano. Como derecho social la educación es fundamental para la realización del ser humano, en la cual se armonizan sus facultades para participar en el desarrollo social. Ello dio origen a que la educación se norme como derecho desde los pactos internacionales y que los Estados los adhieran en sus políticas.

La normatividad del derecho a la educación en los Estados, se concretiza en la práctica social, cobrando importancia en la vida cotidiana, siendo este espacio donde los conceptos de justicia, equidad e igualdad, se entretejen en el derecho a la educación. En este sentido el posicionamiento que tiene Bolívar (2005) sobre la equidad e igualdad de la educación aclara sobre la relación que se guardan los conceptos, él plantea que cuando la equidad y la justicia de la educación es pensada para los alumnos que presentan una situación, bien sea de origen, biológico o social, la justicia se considera distributiva, la cual muchas veces es impuesta, pues no logra satisfacer las demandas para quienes va dirigida la enseñanza, él fundamenta lo que dice con la cita que obtiene de Dubet y DuruBellat (2004) y, Dubet (2004b) sobre la educación compensatoria que se da a estos alumnos, diciendo que lo compensatorio lleva por tanto limitaciones para favorecer la educación de los alumnos:

a) estos dispositivos, como enseña la experiencia, siempre tienen una influencia limitada y no consiguen alterar de modo sensible el juego de la producción de desigualdades escolares;

b) la justicia distributiva encuentra siempre fuertes resistencias por parte de los defensores del modelo meritocrático para asegurar eficazmente la reproducción de sus ventajas competitivas;

c) los grupos sociales peor posicionados, que serían los que debieran defender esta orientación, no suelen estar capacitados para hacer oír su voz y defender las políticas compensatorias (Dubet y Duru-Bellat, 2004:109; Dubet, 2004b:546, en Bolívar, 2005, p. 48).

En el apartado del inciso c), que plantean los autores citados por Bolivar, se desprende que al ser los grupos sociales peor posicionados quienes debieran defender su posición y no lo hacen, se considera entonces la condición cultural a la que han sido proclives de formación los profesores. En tanto los alumnos que son atendidos por ellos se ajustan a lo que las ideas de ellos les dan.

La condición cultural entonces, hace que la educacion compensatoria, que pudiera nombrarse de adecuación a las circunstancias en las que se encuentran, se limita a lo que los profesores reconocen dentro de la posición en que se mantienen dentro del rol de la formacion de los alumnos. Al llevar al terreno de experiencia docente este posicionamiento de los autores que cita Bolívar ¿Qué significa para los profesores que realizan su enseñanza en contextos vulnerables? ¿Cómo han atendido la igualdad, la equidad, la justicia en el derecho a la educación?, ¿Cómo han atendido el derecho a la educación de la población migrante extranjera?

La justicia en tanto, para los menos favorecidos debe considerar una igualdad de oportunidades, por citar lo que plantea Rawls citado por Bolívar

"suponiendo que haya una distribución de dotaciones innatas, los que tienen el mismo nivel de talento y habilidad y la misma disposición a hacer uso de esos dones deberían tener las mismas 
perspectivas de éxito independientemente de su clase social de origen, la clase en la que han nacido y crecido hasta la edad de la razón. En todas las partes de la sociedad debe de haber aproximadamente las mismas perspectivas de cultura y logro para los que están similarmente motivados y dotados" (Rawls, 2002: 74, en Bolívar, 2005, p. 53).

La justicia como igualdad equitativa de oportunidades, independiente a cuales sean las condiciones de origen de las personas en la estructura social, es en el planteamiento de Rawls (2002, en Bolívar, 2005) una proposición de reconocer la justicia que considera las capacidades de las personas, en igualdad. Bolívar dice que un "sistema educativo será más equitativo que otro si las desigualdades existentes en el ámbito educativo son ventajosas para los más desfavorecidos" (Bolívar, 2005, p. 55).

\section{Igualdad equitativa de oportunidades}

Posicionar a los que menos tiene en igual de oportunidades a los que nacieron teniendo, esto incluye a los que por su origen biológico o social son relegados, de tal manera que al proponer una educación compensatoria que busque su igualdad y equidad, y que se aleje de paliativos que se dan para que se adapten a las demandas sociales que se solicitan para todos, sería abrir la brecha existente de inequidades.

Contextualizando el derecho a la educación desde este planteamiento teórico de Rawls en Bolivar, a investigaciones sobre los derechos de alumnos inmigrantes a la educación, hay estudios en México que presentan realidades sobre cómo están accediendo a este derecho. La investigación realizada en Michoacán por Méndez Puga, Castro Valdovinos y Vargas Silva (2011) con alumnos migrantes jornaleros, da cuenta de cómo a partir de un proyecto de investigación acción, alumnos y profesores participan conjuntamente para que los alumnos vean la lecto-escritura con funcionalidad social.

La investigación plantea las acciones que desarrolló el Programa de Educación Básica para Niños y Niñas de Familias Jornaleras Agrícolas Migrantes (PRONIM), en tanto propuestas con estos alumnos inmigrantes jornaleros, acordes a sus necesidades educativas que les permitiera aprender dentro y fuera de la escuela. Las consideraciones a las que llegan los autores, es que el proceso en el que se encuentran estos niños es complejo, por las siguientes características: se mueven constantemente de lugar, sus padres están más enfocados a que apoyen a la economía a que vayan a la escuela (los hijos apoyan con mano de obra), en otros casos los hijos más grandes (principalmente las mujeres) se quedan al cuidado de los hermanos menores, entre otros.

Al posicionamiento de los autores, relacionándolo con lo que plantea Rawls sobre el derecho a la educación, le hace falta enfatizar que el derecho a la educación comparte un todo, que el aprendizaje de la lecto-escritura es una parte del acceso a bienestar social como el que plantea Azúa Reyes, sin embargo en esta investigación de Méndez Puga, Castro Valdovinos y Vargas Silva (2011), se da un enarbolamiento a lo que PRONIM desarrolló con la población menor migrante, por lo que solo esquematiza una parte de lo que establece el plan de estudios, desde otro plano es dar a conocer lo que pudiera considerarse como lo loable de la puesta en práctica de un proyecto de gobierno en atención a la población que se desplaza de su lugar de origen para proveerse de ingresos económicos.

Van Dikj (2006), en el estudio que realiza con alumnos migrantes en Guanajuato en la parte de la sierra, al igual que Méndez Puga, Castro Valdovinos y Vargas Silva 
(2011), situa el aprendizaje de la lecto escritura y las matemáticas en la formación de estos alumos. Su investigacion la realiza en 12 comunidades rurales de ese Estado con altos ínidices migratorios. Las conclusiones a las que llega la autora, es que

\begin{abstract}
A la luz de la Convencion Internacional de los Derechos del Niño, la escuela no está cumpliendo con los critrerios acordados internacionalmente, pues no logra introducir eficazmente los elementos básicos de la cultura que dan acceso a la información y los conocimientos generados internacionalmente, no hay pertinencia con el entorno cultural, no logra el aprendizje necesario para que las nuevas generaciones se inserten a la vida productiva de manera propositiva y con oportunidades reales de alcanzar niveles que trasciendan la pobreza (2006, p. 62).
\end{abstract}

Considerar la formación de todos los alumnos, en específico a los alumnos que viven en alto grado de migración permite identificar qué se está haciendo en su formación. Cómo el derecho a la educación que tiene les da incentivos para sobre-vivir en espacios inciertos donde se consideran victimas de lo que les pasa, como plantea Van Dijk. Por consiguiente la formación de los alumnos migrantes ¿cómo se piensa por parte de los profesores que los atiende?, ¿qué es para ellos atenderlos?, ¿cómo los significan? $\mathrm{Y}$ ¿qué experiencias docentes ha tenido como profesionales de la formación de estos grupos de personas flotantes y que tienen derecho a la educación donde su capacidad no es limitante para que accedan a los bienes sociales como plantea Rawls?

Tinley (2006), por su parte hace un estudio desde el corte cualitativo donde da cuenta de la experiencia educativa que han tenido cuatro familias mexicanas como parte de la migración a nuevos destinos de Estados Unidos. El estudio gira entorno:

Al papel que juega la enseñanza en la vida de los hijos y de qué manera se transforma al cruzar la frontera. [Comenta que] las familias migrantes suelen enfrentar un contexto de discriminación y hostilidad que afecta el proceso de aprendizaje de los hijos y que suele llevar al abandono de la escuela (Tinley, 2006, p. 143).

La realidad que se presenta en este estudio, deja ver que las hostilidades de parte de los receptores de los migrantes de nación a nación, es una posición de racionalidad política de quien hace la aceptación, por lo tanto la situación cultural se ve involucrada en tal postura, que quienes hacen el recibimiento contravienen las normas legales de los pactos $\mathrm{y}$ acuerdo internacionales que firman los paises receptores (aunque Estados Unidos no lo ha hecho), según el estudio teórico que realiza Azúa Reyes (1987) en el Derecho a la educación, en la acción el derecho aun no se cumple, lo cultural de los contextos incide en las prácticas que se realizan en el proceso de ejercer el derecho.

Tinley, en su trabajo llega a utilizar el concepto de 'mejores prácticas', refiriendose por ello a que "el concepto de mejores prácticas para señalar cómo es posible transformar las posturas educativas tradicionales, en oposición al concepto de pensamiento deficiente, el cual adjudica el 'fracaso' del proceso de aprendizaje a los alumnos y sus familias" (2006, p. 143).

La investigación de Tinley, da un planteamiento de las experiencias de las familias migrantes de Guanajuato y su vida en los Estados Unidos, las situaciones de discriminación y marginación a la que se ven expuestos, permite reconocer que el país receptor dentro de su demagogia democrática que dice, hay en la cotidianidad otro 
argumento de cómo se trata al migrante, pues se le considera otro, el que llegó, el no invitado. ¿qué pasa entonces en la frontera sur de México donde las condiciones de vida son criticas económicamente para el propio mexicano, y para el centroamericano? ¿Cuáles serán las experiencias de las familias centroamericanas en la atención de la educación que reciben sus hijos? ¿el profesor que atiende a la población migrante centroamericana qué experiencias tiene en la atención de ellos?

Aguilar Sahagún (2011), en un ensayo que realiza, analiza a la educación como derecho, centrandose en ello como objeto de estudio, plantea que

\begin{abstract}
"la percepcion de la educacion como un bien indiviudal es muy reciente. Su consignación como derecho estaba más relacionada con su concepción como un bien par el Estado, más que para el individuo. Plantea que cuando en el siglo XX se declaró que la educación es un derecho humano, su caracterización como un bien del individuo fue considerado como un medio, ya sea para el pleno desarrollo de la persona, ya para tener acceso a otros bienes sociales. A ese medio se refiere la igualdad de oportunidades, que parece caracterizar lo propio de la justicia educativa" (2011, p. 99).
\end{abstract}

La postura que presenta Aguilar Sahagún, refiere al rol que toma el individuo en la educación, a partir de la declaración como derecho humano. Se involucra las ventajas que obtiene el individuo al ser el centro de desarrollo y no el Estado como tal. Las implicaciones entonces están en miras de una formación de ser humano que pueda acceder a otros bienes sociales, por lo que su planteamiento analiza la educación como igualdad de oportunidades y como justicia en la formación del individuo.

Reconocer entonces lo hecho en legislación educativa, es reconocer los derechos que se han normado desde el exterior del Estado en beneficio de los individuos. Por lo que Pacheco (2010) al abordar la situación del Derecho a la educación en México. Discurso y realidad, en el que analiza los rasgos de sentido y significado sobre el derecho a la educación, comenta que: significados presentes en la configuración del discurso sobre el "derecho a la educación" provenientes de agencias y actores internacionales, nacionales, jurídico normativos $\mathrm{y}$ también, los originados bajo el amparo de las ciencias sociales. La finalidad es valorar hasta qué punto y en qué términos, el recurso discursivo en educación puede aspirar a orientar no solo el sentido sino también las acciones en materia de "derecho a la educación" en México" (Pacheco Méndez, 2010, p. 234).

Ello entonces permite reconocer que la educación como derecho y como justicia al individuo desde el planteamiento de Aguilar Sahagún, se complementa al decir Pacheco Méndez, cómo se reconfigura el derecho en el sentido de poder orientar las acciones dentro del proceso de formación. Implica por tanto ver en la acción cómo ese derecho se cumple, y cómo se ha significado a partir de la experiencia en los profesores.

El cuestionamiento tiene que ver a partir de que Pacheco Méndez comenta que en México el derecho a la educación gira en torno a cuatro ejes: "la proveniente de espacios internacionales, la concerniente a la política nación-gobierno, la jurídico-política y por último, la sociológica” (2010, p. 235). Las bases en que se sostiene su postura la llevan a revisar esos apartados, y los relaciona dentro de lo dado dándose en el proceso de las acciones del derecho a la educación.

Plantear entonces el derecho a la educación resulta no un mero hacer desde la 
idea del profesor sino desde la manera en que subjetiviza al sistema como organizador de la vida escolar a partir de la legislación educativa que realiza, por lo tanto la acción que el profesor realice de dar el derecho a la educación de los alumnos, es en función de dos situaciones. La primera de lo dado como norma para que el derecho a la educación se cumpla, el segundo desde la acción de cómo se lleva a cabo ese derecho a la educación.

Leco Tomas, Kido Cruz y Molina Martínez (2008), en la investigación que realizan sobre Educación, migración e indígenas. Purhépechas en Burnesville, Norte de Carolina, analizan el grado de escolaridad de los migrantes, a partir de las etapas históricas de la migración, por generación, su grado de escolaridad, sus experiencias en la escuela, los impactos y sus perspectivas en torno a la educación en el marco del circuito migratorio internacional México-Estados Unidos.

\section{Cómo se lleva a cabo ese derecho}

La investigación la enfocan a cómo se dan los procesos de migración de estos ciudadanos mexicanos en México y la manera en que se sitúan como migrantes en los Estados Unidos. Los autores dicen que en estos espacios de indígenas mexicanos la migración local se da por:

"La falta de empleos en la región, los bajos salarios, la veda por el bosque, lo poco redituable que resulta labrar la tierra, la falta de mercado para ofertar sus productos artesanales, pero sobre todo la manera en que se han ido fortaleciendo las redes sociales de la migración que forman la cultura local" (Leco Tomás, Kido Cruz, y Molina Martínez, 2008, p. 110).

Las razones que motivan a los indígenas a moverse e incluso ver a la migración como cultura, redunda en su escolaridad y sus aspiraciones en ambos lados de la frontera México- Estados Unidos. Sin embargo la situación se tensa al plantearles un reto la lengua, la edad, la adaptación al nuevo ambiente, entre otros factores. Más, las condiciones de género, hombres y mujeres más proclives en el lado mexicano, en el lado estadounidense los hombres enfocados a la producción económica y las mujeres más enfocadas al término de estudios, quienes están alcanzando una mayor escolaridad.

Lo que estos autores encuentran en su investigación, aporta conocimiento sobre el proceso historico al que se han enfrentado los migrantes mexicanos y su acceso a la educacion, sin embargo en el estudio realizado en esa comunidad indigena hay otras situaciones como las citadas arriba que permean para que la migracion se mantenga hacia los Estados Unidos, con la salvedad que su interés se empieza a centrar en que sus hijos tengan una escolaridad.

La investigacion anterior, lleva a plantear el cuestionamiento sobre los inmigrantes centroamericanos y la educación que reciben en México. Dado que la situación de los migrantes centroamericanos en al frontera sur de México, pareciera no distar de la que viven los mexicanos en los Estados Unidos, que pudiera pensarse que porque en esta parte de la frontera sur, no existe una lengua de dominio económico mundial que sea impedimento para que ellos se incluyan en las aulas y accedan al derecho a a la educación, sí se encuentran significatividades en cuanto a su permanencia, acceso, lengua, entre otros. Reconociendo que si bien hay condiciones económicas mejores en Estados Unidos que en México, hay razones similares por la que las personas centroamericanas hacen una estancia de vida en México o se quedan definitivmente. 
En otra perspectiva, el gobierno mexicano ha creado el Programa Binacional de Educación Migrante, que nace en el Estado de Michocán con la idea de apoyar a las familias que continuamente se mueven entre México y los Estados Unidos (Leco Tomás, Kido Cruz, y Molina Martínez, 2008), más Rojas Wiesner (2010), cita que este programa no solo está dirigo a atender la necesidades de eduativas de niños y niñas emigrantes e inmigrantes México-Estado Unidos, sino que incluye a niños y niñas inmigrantes de Centroamérica. Hace la aclaracion que la poblacion inmigrante centroamericana ha sido objeto de atención desde el 2008, más, agrega que en esta parte de la frontera Sur los objetivos del programa se encuentra en proceso, hay desconocimiento de la dinámica migratoria y al tipo de población que se quiere atender.

\section{La situación compleja en el acceso}

Sin embargo comenta que el Programa de Educación Básica para Niños y Niñas de Familias Jornaleras Agrícolas Migrantes (PRONIM) empieza a operar en el 2008 en Chiapas para atender a la población inmigrante en edad escolar. Comenta que el calendario escolar para estos alumnos es modificado del calendario escolar oficial. Por otro lado el PRONIM está enfocado atender a niños y niñas migrantes que se encuentran en campamentos agricolas. En el contexto del Soconusco, Chiapas, el programa se desarrolla prinicpalmente en las fincas cafetaleras. La aparicion del programa es derivado principalmente de los:

"derechos sociales, económicos y culturales, fundamentalmente, a los que como país, México se ha comprometido a velar y a garantizar el haber firmado convenios y tratados internacionales, pero a los que tambien está obligado a salvaguardar por tratarse de derechos fundamentales, como el de la educación y la salud" (Rojas Wiesner, 2010, p. 152).

En la investigación, Rojas Weisner da a conocer cómo se da el acceso a la educacion en la población de alumnos migrantes. Considerando lo anterior, la situación se torna compleja en el acceso que tienen los alumnos a la educación, principalmente en cuanto a su condición migratoria irregular, las condiciones de vida y el trabajo que desempeñan como migrantes temporales $y$ residentes transfronterizos, que afectan su acceso y permanencia a la educación en tal dinámica que se encuentran.

En otro ángulo, la investigación que realiza Leal Sorcia (2011), permite comprender cómo son las escuelas que se ubican en campamentos y fincas, donde niños y niñas migrantes acceden a servicios educativos de nivel básico, en dos regiones contrastantes: el noroeste del país, donde se localizan campamentos de agroexportación, y la región del Soconusco, Chiapas, en la frontera sur, específicamente en fincas cafetaleras.

Del estudio se toma el interés que el PRONIM ha tenido para que estas poblaciones accedan a la escuela, que para efectos de esta investigación es en la población guatemalteca instalada en las zonas cafetaleras. Se hace mención que para el caso de los Estados de la república mexicana donde haya presencia de jornaleros migrantes se adecúa un calendario escolar de tal manera que los alumnos continúen con sus estudios.

En el caso de la investigación que se presenta, los profesores que han atendido a alumnos provenientes de Centroamérica, no corresponde a la de los jornaleros agrícolas, las condiciones laborales de los padres de esta población se desarrolla en la informalidad dentro del cuadro de la ciudad (de ello hablaré en otro capítulo), por lo que son atendidos por profesores que han terminado una licenciatura, el sistema educativo actualiza la 
profesión docente con cursos o diplomados, además que se tienen otros derechos como trabajador de base al servicio del Estado mexicano, totalmente diferente a los profesores que atienden a los alumnos en los campamentos y fincas agrícolas.

Las características de los profesores que laboran en los campos agrícolas es que son jóvenes que han terminado la educación secundaria, media superior o en algunos casos son jóvenes que se encuentran estudiando una licenciatura a fin a la educación o tienen licenciatura trunca. Sus actualizaciones están basadas a capacitación por instructores, reciben alimentación por parte de quien los contrata y su pago es menor a lo que un profesor en la escuela regular percibe.

El estudio de Leal Sorcia por tanto reconoce los esfuerzos que hace PRONIM en la atención a la población migrante en el acceso a la educación básica, sin embargo nacen cuestionamientos cómo, ¿qué ha significado para los profesores el trabajar con estos alumnos?, para los profesores que los atienden en las escuelas regulares que trabajan de acuerdo a calendario escolar oficial ¿cómo han vivido su quehacer con alumnos migrantes, una vez que PRONIM, no está enfocado a ellos?

La situación actual del estado de la cuestión sobre el acceso a la educación de la población migrante en México-Estados Unidos, como la que se ha escrito MéxicoCentroamérica, da un planteamiento de cómo la lengua en el primer caso es un detonador para que se encuentren en desventaja los alumnos que van de México y estudian es Estados Unidos, así como los que estudian en Estados Unidos y vienen a México a continuar o concluir estudios.

Los retos son grandes, pues ni los Estados Unidos preparan a los alumnos migrantes para el retorno a México, ni México los prepara para ir a Estados Unidos, aunque se han hecho convenios por ambos países, aún resulta poco, dada las condiciones en que los mexicanos se encuentran por allá. Para el caso de mexicanos que retornan por un tiempo o definitivo a México la situación tampoco es alentadora, de las investigaciones revisadas, se constata que los profesores mexicanos tampoco los pueden tratar.

En relación a la educación que se da en el Soconusco, Chiapas, México, las investigaciones muestran el apoyo que ha dado PRONIM a las familias jornaleras agrícolas, se aclara que la atención a esta población en Chiapas empezó a operar en el año 2008, ello resulta interesante pues son seis años (hasta 2014) que lleva operando, requiere de una evaluación de qué resultados ha obtenido y de qué manera, sin embargo en este momento esa idea queda guardada, porque la investigación que se realiza y por la cual se revisa el conocimiento escrito sobre la educación de la población migrante MéxicoEstados Unidos y México-Centroamérica, se enfoca a la educación que se da a la población migrante que se encuentra inscrito en las escuelas regulares donde el calendario escolar inicia en agosto y termina en julio, más los profesores que los atienden tienen una formación profesional de licenciatura y que reciben actualización docente permanentemente.

El Programa Binacional de Educación Migrante en coordinación con el PRONIM en esta área de la frontera sur, ha impulsado el acceso a la educación de la población en condición de migración, resulta por tanto pertinente cuestionar ¿cómo se relacionan estos programas con los profesores que están en las escuelas regulares para trabajar con ellos en atención de estos alumnos?, además que ello lleva a plantear el cuestionamiento ¿cómo hacen el quehacer docente los profesores que atienden a los alumnos inmigrantes que no están en las fincas cafetaleras?, ¿cómo piensan que hacen la 
inclusión educativa?, ¿qué experiencias han tenido de ello?

Lo encontrado en el estado de la cuestión desde la inclusión que se hace de alumnos que presentan capacidades diferentes por su condición física o cognitiva, y social, más las investigaciones que se hacen sobre la educación de los migrantes mexicanos en Estados Unidos, la atención que ha tenido el gobierno mexicano hacia ellos cuando regresan a México, la educación que se da a la población migrante jornalera agrícola en México, y la atención educativa que ha habido en México a la población migrante proveniente de Centroamérica, se plantea la siguiente pregunta ¿cómo configuran sus experiencias docentes los profesores en los procesos de inclusión de niños y niñas inmigrantes centroamericanos en las escuelas primarias públicas de Tapachula, Chiapas?

Con base a lo revisado, se plantean la pegunta principal y las preguntas secundarias que guiarán la investigación.

Pregunta principal

¿Cómo configuran sus experiencias los docentes en los procesos de inclusión de niños y niñas inmigrantes centroamericanos en las escuelas primarias públicas de Tapachula, Chiapas?

Preguntas secundarias

- ¿Cómo se ha caracterizado el derecho a la educación básica de la población migrante extranjera en México?

- ¿Cómo han sido los procesos de formación de los docentes respecto a la inclusión educativa?

- ¿Qué experiencias tienen los docentes respecto a los procesos de inclusión educativa de niños y niñas inmigrantes?

- ¿Qué prácticas pedagógicas han considerado los docentes para promover la inclusión educativa de niños inmigrantes a partir de la experiencia docente?

Las preguntas de investigación dan paso a establecer los objetivos de la investigación. Los cuales se dividen en general y específicos, quedando establecidos de la siguiente manera.

\section{General}

Analizar la configuración de la experiencia docente de los profesores de educación primaria del municipio de Tapachula; Chiapas, respecto a los procesos de inclusión educativa de niños y niñas inmigrantes de Centroamérica.

\section{Específicos}

Caracterizar la migración centroamericana en la región del Soconusco, Chiapas, en su derecho y atención a la educación básica.

Indagar los procesos de formación de los docentes respecto a la inclusión educativa en Tapachula, Chiapas.

Reconocer la importancia de las experiencias docentes en los procesos de inclusión educativa de niños y niñas inmigrantes provenientes de Centroamérica.

Caracterizar las prácticas pedagógicas que construye el docente para atender los planteamientos de la inclusión educativa a partir de su experiencia con niños y niñas inmigrantes provenientes de Centroamérica.

\section{Consideraciones finales}

La construcción de las preguntas y los objetivos de la investigación presuponen que la experiencia de los docentes sobre los procesos de inclusión de niños y niñas 
inmigrantes centroamericanos en las escuelas primarias públicas de Tapachula, Chiapas, se configuran en el sentido de que no están preparados para atender la diversidad cultural para la inclusión, pues la realizan desde lo que su cotidianidad les ha ofertado.

\section{Bibliografía}

1. Aguilar Nery, J. (2004). Hacia una Memoria Argumental sobre la educación Intercultural en México. Una narrativa desde la frontera Norte. Revista Mexicana de Investigación Educativa, 39-59.

2. Aguilar Sahagún, L. A. (2011). La educación como objeto de derecho. Revista Latinoamericana de Estudios Educativos (México), vol. XXI, núm. 4, 99-110.

3. Aja, E. (2000). La inmigración extranjera en España. Los retos educativos. Barcelona: Fundacion "La Caixa" .

4. Alcántara Santuario, A., \& Navarrete Casales, Z. (2014). Inclusión, equidad y cohesión social en las políticas de educación superior en México. Revista Mexicana De Investigacion Educativa, 19, 60, 213-239.

5. Ayala Carabajo, R. (2011). La mirada pedagógica : una mirada fresca y profunuda a la experiencia educativa desde el enfoque de van Manen. Revista Española de Pedagogía, 119-144.

6. Azúa Reyes, S. T. (1987). Los derechos humanos y el derecho a la educación. Revista de la Facultad de Derecho de México. Tomo XXXVII, Núms. 154-155-156, 361-375.

7. Bolívar, A. (2005). Equidad educativa y teorías de la justicia. Revista Iberoamericana sobre Calidad, Eficacia y Cambio en Educación, vol. 3, núm. 2, 42-69.

8. Cárdenas Leitón, H. (2011). Referentes teóricos y metodológicos de los docentes en servicio con una perspectiva de inclusión educativa: creencias y prácticas. Revista electónica "Actualidades investigativas en educación", 1-38.

9. Casado Muñoz, R., \& Lezcano Barbero, F. (2012). Educación en la escuela inclusiva. Formación del profesorado y perspectiva de futuro. Argentina: Magisterio del Río de la Plata.

10. Constitución Política de los Estados Unidos Mexicanos. (2013). Cámara de Diputados. Diario Oficial de la Federación.

11. Delgado Montoya, W. (2007). Inclusión: principio de calidad educativa desde la perspectiva del desarrollo humano. Revista Educación 31(2), 45-58.

12. Doin, G. (Dirección). (2012). La educación Prohibida [Película]. 13. Fontana, A., \& Urquiza, P. (2011). La igualdad en la escuela primaria. Una experiencia de política socio-educativa. Los centros de actividades infantiles. En M. G. Vallone, La educación como factor de 
inclusión social: Debate iberoamericano. Madrid: Dykinson.

14. Kymlicka, W. (2006). Fronteras territoriales. Madrid: Minima Trotta.

15. Leal Sorcia, O. (2011). Escuelas en campamentos y fincas en México: experiencias educativas con niños jornaleros migrantes. Revista Electrónica de Psicología Iztacala. Vol. 14, Núm. 2, 310-334 http://www.iztacala.unam.mx/carreras/psic ologia/psiclin/vol14num2/Vol14No2Art17. pdf.

16. Leco Tomás, C., Kido Cruz, A., \& Molina Martínez, R. (2008). Educación, Migración e Indígenas. Purhépechas en Burnesville, Norte Carolina. Revista Nicolaita de Políticas Públicas. CIMEXUS. Vol. 3, núm. 1, 107129.

http:// cimexus.umich.mx/index.php/cim1/ article/view/28.

17. López Melero, M. (2004). Construyendo una escuela sin exclusiones: Una forma de trabajar en el aula con proyectos de investigación. Archidona, Málaga: Ediciones Aljibe.

18. Martínez, M. (2011). ¿Es el multiculturalismo bueno para los migrantes? Is Multiculturalism Good for Inmigrants? Reis 135, 27-46. doi:10.5477/cis/reis.135.27. 19. Méndez Puga, A. M., Castro Valdovinos, I. L., \& Vargas Silva, A. D. (2011). "Yo también tengo derechos". Procesos educativos en campamentos de familias jornaleras migrantes en Michoacán. XI Congreso Nacional de Investigación Educativa / 1. Aprendizaje y Desarrollo Humano / Ponencia.

20. Montes, N., \& Ziegler, S. (2010). Miradas sobre una experiencia de cambio en la escuela secundaria. Nuevos formatos para promover la inclusión educativa. Revista Mexicana de Investigación Educativa. OctubreDiciembre, Vol. 15, Núm. 47, 1075-1092. 21. Opertti, R. (20 de 03 de 2014). La educación inclusiva, según Renato Opertti y Carlos Skliar. Obtenido de http://www.ucm.edu.co/: http://www.ucm.edu.co/2014/03/laeducacion-inclusiva-segun-renato-opertti-ycarlos-skliar/

22. Organización de las Naciones Unidas para la Educación, la Ciencia y la Cultura. (1994). Declaración de Salamanca y Marco de Acción para las necesidades Educativas especiales. París: Ministerio de Educación y Ciencia España. (ED-94/WS/18).

23. Pacheco Méndez, T. (2010). Derecho a la educación en México. Discurso y realidad. Revista de la Asociación de Sociología de la Educación. www.ase.es/rase, vol. 3, núm. 2, 234-248.

24. Rojas Wiesner, M. L. (2010). Migración y educación en regiones fronterizas. El caso de 
los migrantes centroamericanos en Chiapas, México, un tema pendiente. Educación Superior y Sociedad, Vol 15, No 2, 133-162.

25. Tinley, A. (2006). Migración de Guanajuato a Alabama. Experiencias escolares de cuatro familias mexicanas.
Sociológica, vol. 21, núm. 60, enero-abril, 143-172.

26. Van Dikj, S. (2006). Migración, escula y niñez. Revista Regional de Investigación Educativa, 49-66.. 